\title{
Short Communication on: Study of Avidity of Antigen-Specific Antibody as a Means of Understanding Development of Long-Term Immunological Memory after Vibrio cholerae O1 Infection
}

\author{
Mohammad Murshid Alam, Taufiqur Rahman Bhuiyan and Firdausi Qadri* \\ Infectious Diseases Division, International Centre for Diarrhoeal Disease Research, Dhaka, Bangladesh \\ *Corresponding author: Firdausi Qadri, Infectious Diseases Division, International Centre for Diarrhoeal Disease Research, Dhaka, Bangladesh, E-mail: \\ fqadri@icddrb.org
}

Received date: June 19, 2017; Accepted date: July 20, 2017; Published date: July 25, 2017

Copyright: (2017 Alam MM, et al. This is an open-access article distributed under the terms of the Creative Commons Attribution License, which permits unrestricted use, distribution, and reproduction in any medium, provided the original author and source are credited.

\section{Short Communication}

Interaction between antigen and antibody modulates key biological processes in neutralizing and eliminating pathogenic microorganisms following infection. The strength of this interaction depends on the affinity, valency and three dimensional arrangements of the interacting epitopes and paratopes. The measure of the overall strength of this multimeric interaction is termed as 'Avidity'. Development of antibody with high avidity is a crucial feature of a secondary immune response followed by a low avidity antibody producing primary response $[1,2]$. Thus antibody avidity has been used as a marker for B cell maturation and is an important surrogate for determining long term immunological memory responses to pathogens. In our article entitled "Study of avidity of antigen-specific antibody as a means of understanding development of long-term immunological memory after Vibrio cholerae O1 infection" we showed that antibody avidity after infection and immunization is a good correlate of the development and maintenance of memory $\mathrm{B}$ cell responses to $V$. cholerae $\mathrm{O} 1$ antigens. To the best of our knowledge this is the first study that assesses antibody avidity following a non-invasive mucosal infection [3].

$V$. cholerae infection still remains a significant threat for humans residing or travelling to the regions of the world with limited resources for clean water and adequate sanitation. Naturally occurring cholera infection is known to provide protection against the disease for several years, although the currently available licensed oral cholera vaccines fails to induce comparable protective immunity especially in young children. Therefore, understanding the mechanism of protection against cholera is crucial for the development of an effective vaccine.

Our study showed that individuals immunized with a killed whole cell cholera vaccine (Dukoral) did not generate immune response comparable to the patients $\mathrm{d}$ following a severe cholera requiring hospitalization. Cholera patients showed a longer persistence of IgG and IgA antibodies of high avidity to both $\mathrm{T}$ cell dependent antigen, cholera toxin as well as the $\mathrm{T}$ cell independent antigen lipopolysaccharide (LPS). This was compared to the vaccinees. LPS is considered as a major protective antigen for $V$. cholerae and thereby antibodies with high avidity directed against the LPS play a crucial role in protection [4]. In young children both LPS and to the antigenic component of the LPS, the O-specific polysaccharide (OSP), specific immune response is poor following natural infection. High avidity antibodies to LPS can provide protection by inhibiting the motility of $V$. cholerae strains which can prevent a successful colonization. Generation and long term persistence of LPS specific antibodies with high avidity in patients infected with cholera may explain in part that protective mechanism develops in naturally occurring cholera. The lack of such responses in vaccinees thus can account for the lack of long term protection in these individuals. Similarly, longer persistence of cholera toxin specific antibodies with high avidity has the potential to neutralize the toxin more efficiently and can play a crucial role in the pathogenesis of cholera.

Memory B cell responses is a measure for studying long lasting immunity against infection. Upon exposure to an antigen, memory $B$ cells can be generated in the germinal centre of the secondary lymphoid organs and can provide protection by differentiating into high affinity antibody producing plasma cells after re-exposure to the same pathogen [5]. In our study we showed that cholera patients developed both CTB and LPS specific IgA and IgG memory B cell responses whereas the vaccines only showed increase in memory $B$ cell responses to CTB but not to LPS which can explain in part, why the killed whole cell vaccine used in the study is not as effective as naturally occurring cholera. A significant finding of this study is that the memory B cell responses to both CTB and LPS correlate significantly with the avidity indices of the corresponding antibodies. This suggests that, generation of antigen specific antibodies with high avidity may be occurring as a part of B cell proliferation. During this process the subset of $B$ cells producing antigen specific antibodies with high avidity will differentiate and generate memory B cells that can lead to immunity against cholera which can protect individuals from future infection.

In resource limited settings especially in those regions of the world where cholera and other infectious diseases are endemic, measuring antigen specific memory B cell responses using cell culture followed by Enzyme-linked immunosorbent spot (ELISPOT) technique is challenging. Measuring avidity of antibodies on the contrary is a simpler, quicker and cheaper technique performed by Enzyme-linked immunosorbent assay (ELISA) using serum samples. This response can also be measured in stored samples compared to the memory B cell responses where live cells are used. Measuring antibody avidity also require a small amount of blood compared to the cell culture based ELISPOT assay which could be advantageous in measuring Long-term immunological memory response following infection or vaccination in children. Therefore, to assess the progression of immunity in patients as well as measuring the development of memory responses in vaccinated individuals in resource limited settings, antibody avidity has the potential to serve as a good surrogate for immunological memory.

Antibodies with high avidity have been shown to be more potent than antibodies with low avidity in bactericidal assays for Haemophilus influenzae and Streptococcus pneumoniae [6,7] suggesting that antibody avidity could be an appropriate marker for 
Citation: Alam MM, Bhuiyan TR, Qadri F (2017) Short Communication on: Study of Avidity of Antigen-Specific Antibody as a Means of Understanding Development of Long-Term Immunological Memory after Vibrio cholerae O1 Infection. J Vaccines Vaccin 8: 363 . doi: $10.4172 / 2157-7560.1000363$

Page 2 of 2

protective immunity. However, the association between high-avidity antibodies and protection against the disease is yet to be determined specifically for mucosal infections. $\mathrm{T}$ cells, specifically the follicular helper $\mathrm{T}$ cells play a key role in the development of an effective memory response by interacting with $\mathrm{B}$ cells in the germinal center inducing antigen specific B cell maturation and differentiation [8]. Very little is known about $\mathrm{T}$ cells and avidity of antibodies in cholera. Investigating the correlation between $\mathrm{T}$ cell responses and antibody avidity can be considered important in understanding the mechanism of protection against cholera and other infectious diseases. It should be recall that cholera toxin is a potent immunoadjuvant in addition to being an enterotoxin [9]. The toxin and LPS may activate mucosal innate immune responses also [10], and abnormalities in innate immune factors have been repetitively associated with susceptibility to cholera [11,12]. Thus, assessing the correlation between innate immune response and protection following cholera warrant further investigation.

\section{References}

1. Matheus S, Deparis X, Labeau B, Lelarge J, Morvan J, et al. (2005) Discrimination between primary and secondary dengue virus infection by an immunoglobulin $\mathrm{G}$ avidity test using a single acute-phase serum sample. J Clin Microbiol 43: 2793-2797.

2. Fox JL, Hazell SL, Tobler LH, Busch MP (2006) Immunoglobulin G avidity in differentiation between early and late antibody responses to West Nile virus. Clin Vaccine Immunol 13: 33-36.

3. Alam MM, Arifuzzaman M, Ahmad SM, Hosen MI, Rahman MA, et al. (2013) Study of avidity of antigen-specific antibody as a means of understanding development of long-term immunological memory after Vibrio cholerae O1 infection. Clin Vaccine Immunol 20:17-23.

4. Chatterjee SN, Chaudhuri K (2006) Lipopolysaccharides of Vibrio cholerae: III. Biological functions. Biochim Biophys Acta 1762: 1-16.

5. Phan TG, Paus D, Chan TD, Turner ML, Nutt SL, et al. (2006) High affinity germinal center B cells are actively selected into the plasma cell compartment. J Exp Med 203: 2419-2424.

6. Schlesinger Y, Granoff DM (1992) Avidity and bactericidal activity of antibody elicited by different Haemophilus influenzae type b conjugate vaccines. JAMA 267: 1489-1494.

7. Usinger WR, Lucas AH (1999) Avidity as a determinant of the protective efficacy of human antibodies to pneumococcal capsular polysaccharides. Infect Immun 67: 2366-2370.

8. Alam MM, Leung DT, Akhtar M, Nazim M, Akter S, et al. (2013) Antibody avidity in humoral immune responses in Bangladeshi children and adults following administration of an oral killed cholera vaccine. Clin Vaccine Immuno 1 20: 154.

9. Sanchez J, Holmgren J (2011) Cholera toxin-a foe \& a friend. Indian J Med Res 33: 153-163.

10. Ellis CN, LaRocque RC, Uddin T, Krastins B, Mayo-Smith LM, et al. (2015) Comparative proteomic analysis reveals activation of mucosal innate immune signaling pathways during cholera. Infect Immun 83: 1089-1103.

11. Flach CF, Qadri F, Bhuiyan TR, Alam NH, Jennische E, et al. (2007) Broad up-regulation of innate defense factors during acute cholera. Infect Immun 75: 2343-2350.

12. Larocque RC, Sabeti P, Duggal P, Chowdhury F, Khan AI, et al. (2009) A variant in long palate, lung and nasal epithelium clone 1 is associated with cholera in a Bangladeshi population. Genes Immun 10: 267-272. 